\title{
Analysis of Outcomes Using Intraoperative Irrigation and Drainage for Complicated Appendicitis
}

\author{
Monica Sridhar ${ }^{\mathrm{a}, \mathrm{b}}$, Manrique Guerrero ${ }^{\mathrm{a}}$, Jacob Miller ${ }^{\mathrm{a}}$, Paul Hanna ${ }^{\mathrm{a}}$, \\ Jafar Haghshenas ${ }^{a}$, Jamshed Zuberia
}

\begin{abstract}
Background: The practice of irrigation and/or intraoperative peritoneal drain placement during an appendectomy for complicated appendicitis is currently controversial. This study was aimed at delineating the relationship between the practice of intraoperative drain placement and/or irrigation and patient outcomes for complicated appendicitis cases.
\end{abstract}

Methods: A retrospective study of patients presenting with acute complicated appendicitis, which was defined as perforated or gangrenous, was conducted $(n=225)$. The primary outcome was defined as the presence of postoperative abscess or sepsis, while the secondary outcome measured was length of hospital stay.

Results: Patients who received intraoperative drain placement had an increased risk of postoperative abscess (odds ratio $(\mathrm{OR})=13.33, \mathrm{P}<$ $0.001)$ and sepsis $(\mathrm{OR}=11.37, \mathrm{P}=0.026)$. There was no significant difference in primary outcomes with irrigation. Patients who received irrigation had a longer length of stay (7.59 vs. 4.69 days, $\mathrm{P}=0.001$ ) as did those who received an intraoperative drain (7.61 vs. 5.33 days, $\mathrm{P}=0.01)$.

Conclusions: In this study, irrigation did not change the OR of sepsis and postoperative abscess. However, placement of an intraoperative drain increased both the rates of sepsis and postoperative abscesses, indicating possible increase in postoperative complications with intraoperative drains when compared with no drains.

Keywords: Irrigation; Drainage; Complicated appendicitis

\section{Introduction}

Appendicitis, defined as the inflammation of the vermiform appendix, is a common cause of abdominal pain in the emer-

Manuscript submitted July 30, 2019, accepted August 31, 2019

aSt. Joseph's University Medical Center, Paterson, NJ, USA

${ }^{b}$ Corresponding Author: Monica Sridhar, St. Joseph's University Medical

Center, Paterson, NJ, USA. Email: monica.sridhar92@gmail.com

doi: https://doi.org/10.14740/jcs385 gency department and is one of the most common surgical emergencies in the world. Its clinical presentation can vary from simple appendicitis to complicated appendicitis, which is most often delineated in literature as perforated (occurring in about $13-30 \%$ of appendicitis cases [1]) and/or gangrenous appendicitis. Although the management of simple appendicitis is well understood, surgeons debate the use of certain intraoperative surgical techniques when it comes to appendectomies for complicated appendicitis cases.

Current guidelines suggest that perforation in acute appendicitis cases should be managed based on stability of symptoms, with unstable patients receiving immediate appendectomy, and on the presence of a phlegmon or abscess [1]. Intraoperative decisions to place drains and/or irrigate, however, have been a topic of debate in previous studies. These clinical decisions are currently based on surgeon preference. In surgical history, abdominal drainage had conflicting viewpoints. Robert Lawson Tait in the 1800s stated "When in doubt drain!" [2]; however, surgeons such as William Stewart Halsted stated "No drainage at all is better than the ignorant employment of it" [3]. As for irrigation, surgical practice is slowly straying away from the truism "the solution to pollution is dilution" [4].

With debating guidelines, our study aimed to determine the effectiveness of intraoperative drains and/or irrigation in patients who received appendectomies for complicated appendicitis. In the study, complicated appendicitis was defined as perforated and/or gangrenous appendix. We hypothesized that there is a lack of benefit in the use of intraoperative irrigation and/or drainage for patients with complicated appendicitis.

\section{Materials and Methods}

\section{Setting and patient selection}

This study is a single-center retrospective observational study of patients presenting with appendicitis between June 2014 and April 2018 at an academic tertiary care medical center. Data was acquired from the hospital's surgical registry. The study is approved by Institutional Review Board and patient consent was waived due to the observational nature of the study. A total of 225 patients were included in the study, and the patients' age, gender, initial presentation, length of hospital stay, medical management, operative reports, postoperative abscesses, sepsis, white blood cell (WBC), vitals at admis- 
Table 1. Characteristics of Patients Irrigated Versus Not Irrigated

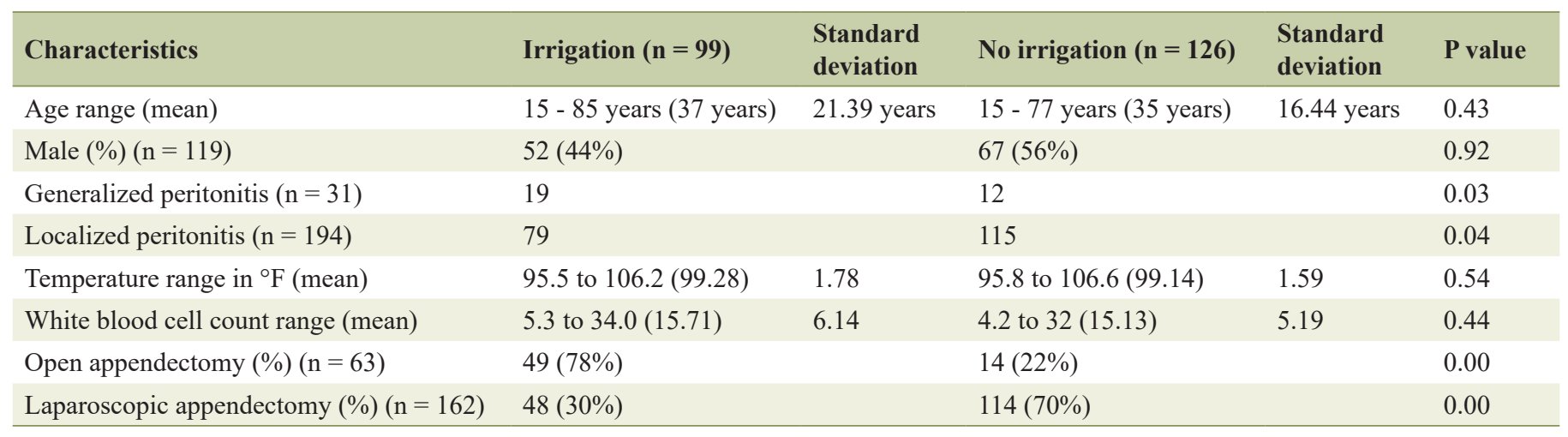

sion, re-intervention, re-admission and mortality status were recorded and analyzed. Operative reports were used to determine the use of intraoperative irrigation and/or drainage for the studied patients. We excluded patients who were younger than 15 years of age, were pregnant, and/or had an initial presentation of an intra-abdominal abscess. The primary outcome was defined as the presence of postoperative abscess and/or sepsis, while the secondary outcome measured was length of hospital stay. Postoperative abscess was defined in the study as a fluid collection that developed postoperatively and confirmed by computerized tomography imaging and by the radiologist's final interpretation of the image. Sepsis in the study was based on patient medical records and billing codes.

\section{Analysis}

All data were quantitative in nature. All data followed a normal distribution and all tests were parametric in nature. Data analysis was performed using Stata 15.1. Univariate and population characteristics were conducted using Student's $t$-test and Pearson's Chi-square test. A P value of $<0.05$ was considered significant. Pairwise correlation was utilized to determine interactions of variables for utilization in regression models. Significance for pairwise correlation was determined as $\mathrm{P}=0.1$. All potential variables were utilized in the development of the models. Multiple linear regression analysis was used to deter- mine length of stay as a secondary outcome and multiple logistic regression analysis was used to evaluate the occurrence of abscess or sepsis as primary outcomes. Analysis was performed for the entire patient population and repeated based off stratification. A P value of $<0.05$ was considered significant.

\section{Results}

\section{Baseline characteristics and initial presentation}

Between 2014 and 2018, there were a total of 225 patients who presented with complicated appendicitis, defined as either having a perforated and/or gangrenous appendix.

Of the 225 patients who presented with complicated appendicitis, 56 patients (about 25\%) were drained, 99 patients (44\%) were irrigated, 45 patients $(20 \%)$ were irrigated and drained, 119 patients (about 53\%) were male, 31 patients (about 14\%) presented with generalized peritonitis, 194 patients (about 86\%) presented with localized peritonitis, 63 patients $(28 \%)$ had an open appendectomy, and 162 patients (72\%) had a laparoscopic appendectomy (Tables 1 and 2).

Of the patients who were irrigated, the median age was 37 years, 52 patients (about 44\%) were male, the average temperature at initial presentation was $37.38^{\circ} \mathrm{C}$, and the average WBC count was 15.71 (Table 1). When comparing patients who were irrigated versus those who were not, there was a sta-

Table 2. Characteristics of Patients Drained Versus Not Drained

\begin{tabular}{|c|c|c|c|c|c|}
\hline Characteristics & Drains $(n=56)$ & $\begin{array}{l}\text { Standard } \\
\text { deviation }\end{array}$ & No drains $(n=169)$ & $\begin{array}{l}\text { Standard } \\
\text { deviation }\end{array}$ & P value \\
\hline Age range (mean) & $15-85$ years (39 years) & 36.63 & $15-83$ years ( 35 years) & 17.77 & 0.19 \\
\hline Male $(\%)(n=119)$ & $38(32 \%)$ & & $81(68 \%)$ & & 0.38 \\
\hline Generalized peritonitis $(n=31)$ & 15 & & 16 & & 0.02 \\
\hline Localized peritonitis $(\mathrm{n}=194)$ & 52 & & 142 & & 0.02 \\
\hline Open appendectomy $(\%)(n=63)$ & $38(60 \%)$ & & $25(40 \%)$ & & 0.00 \\
\hline Laparoscopic appendectomy $(\%)(n=162)$ & $28(17 \%)$ & & $134(83 \%)$ & & 0.00 \\
\hline
\end{tabular}


Table 3. Multiple Regression Analysis for Predictors of Postoperative Abscess

\begin{tabular}{llll} 
& Odds ratio & Confidence interval & P value \\
\hline Drain & 13.33 & $3.75-47.36$ & $<0.001$ \\
Irrigated & 0.636 & $0.242-1.660$ & 0.357 \\
\hline
\end{tabular}

tistically significant difference between those who presented with generalized peritonitis versus those who presented with localized peritonitis and those who had an open appendectomy versus those who had a laparoscopic appendectomy (Table $1)$.

Of those patients who received intraoperative drainage, the median age was 39 years, 38 patients (about 32\%) were male, the average temperature at initial presentation was 37.33 ${ }^{\circ} \mathrm{C}$, and the average WBC count was 16.14 (Table 2). When comparing patients who received intraoperative drainage versus who did not, there was a statistically significant difference between those who presented with generalized peritonitis versus those who presented with localized peritonitis and those who had an open appendectomy versus those who had a laparoscopic appendectomy (Table 2). This finding is similar to that above when comparing patients who were irrigated versus who were not.

\section{Primary outcomes}

When performing a multiple logistic regression analysis for postoperative abscess, there was a statistically significantly increased risk of postoperative abscess for patients who received an intraoperative drain (odds ratio (OR) of 13.33; 95\% confidence interval $(\mathrm{CI}), 3.75$ to $47.36 ; \mathrm{P}<0.001)$ compared to patients who did not receive an intraoperative drain (Table 3 ). However, there was not a statistically significantly increased risk of postoperative abscess for patients who were irrigated (OR of $0.636 ; 95 \% \mathrm{CI}, 0.242$ to $1.660 ; \mathrm{P}=0.357$ ) compared to patients who were not irrigated (Table 3 ).

When performing a multiple logistic regression analysis for postoperative sepsis, there was a statistically significantly increased risk of postoperative sepsis for patients who received an intraoperative drain (OR of $11.37 ; 95 \% \mathrm{CI}, 1.34$ to 96.43; $\mathrm{P}=0.0026$ ) compared to patients who did not receive an intraoperative drain (Table 4). However, there was not a statistically significantly increased risk of postoperative sepsis for patients who were irrigated (OR of $2.86 ; 95 \%$ CI, 0.542 to $15.1 ; \mathrm{P}=0.22$ ) compared to patients who were not irrigated (Table 4).

Therefore, there was a statistically significantly increased risk of postoperative abscess and sepsis for patients who received intraoperative drainage compared to those who did not.

Table 4. Multiple Regression Analysis for Predictors of Sepsis

\begin{tabular}{llll}
\hline & Odds ratio & Confidence interval & P value \\
\hline Drain & 11.37 & $1.34-96.43$ & 0.0026 \\
Irrigated & 2.86 & $0.542-15.1$ & 0.22 \\
\hline
\end{tabular}

Table 5. Length of Stay

\begin{tabular}{llll}
\hline & LOS (days) & Confidence interval & P value \\
\hline No irrigation & 4.69 & $3.51-5.87$ & 0.000 \\
Irrigation & 7.59 & $4.72-10.37$ & 0.001 \\
No drain & 5.33 & $4.26-6.40$ & 0.000 \\
Drain & 7.61 & $4.69-10.47$ & 0.013 \\
\hline
\end{tabular}

LOS: length of stay.

\section{Secondary outcome}

When performing a linear regression analysis for length of hospital stay, there was statistically significant increase in length of hospital stay in patients who received irrigation (7.59 days; $95 \% \mathrm{CI}, 4.72$ to 10.37 days; $\mathrm{P}=0.001)$ compared to those who did not receive irrigation (4.69 days; $95 \mathrm{CI}, 3.51$ to 5.87 days; $\mathrm{P}<0.001$ ) (Table 5). There was also a statistically significant increase in length of hospital stay in patients who received intraoperative drainage (7.61 days; 95\% CI, 4.69 to 10.47 days; $\mathrm{P}=0.013$ ) compared to those who did not receive intraoperative drainage ( 5.33 days; $95 \%$ CI, 4.26 to 6.40 days; $\mathrm{P}<0.001$ ) (Table 5).

Therefore, there was a statistically significantly increased risk of length of hospital stay for patients who were irrigated and received intraoperative drainage compared to those who did not.

\section{Discussion}

Given the fairly prevalent cases of complicated appendicitis, it is important to establish best, evidence-based practices when treating this specific patient population. The use of intraoperative drainage and/or irrigation has been a contested topic in literature and among surgeons. Some surgeons believe that intraoperative drains provide a source of infection by acting as a foreign body and increasing the risk of inflammation [5], potentially increasing the rate of surgical site infections, abscess, and/or sepsis. While other surgeons believe that prophylactic use of intraoperative peritoneal drains prevents the formation of postoperative abscess or sepsis, especially in complicated appendicitis cases.

When it pertains to intraoperative drainage for complicated appendicitis, current literature is controversial. Greenall et al in an 8-year prospective trial published in the British Journal of Surgery established no benefit in the use of intraoperative drain for patients with perforated appendicitis receiving appendectomies [6]. However, Beek et al, in a retrospective study focusing on the effectiveness of peritoneal drainage for patients with perforated appendicitis having surgery, demonstrate a reduction in the overall complication rate for patients receiving intraoperative peritoneal drainage for perforated appendicitis [7]. Similarly, Pakula et al in a retrospective study demonstrated that the use of Jackson-Pratt (JP) drains in patients with perforated or gangrenous appendicitis during laparoscopic appendectomy had decreased rates of pelvic abscess [8]. 
Current literature is also controversial when it pertains to the use of intraoperative irrigation as well. St Peter et al in a prospective randomized trial, although performed in a pediatric population, demonstrated nil benefit in irrigation of the peritoneal cavity over suction alone during laparoscopic appendectomy for perforated appendicitis [9]. Similarly, Hajibandeh et al found in a systematic review that peritoneal irrigation during emergency laparoscopic appendectomy does not provide additional benefits [10]. However, Sun et al in a prospective randomized trial demonstrated that irrigation of the peritoneal cavity status post laparoscopic appendectomy could potentially decrease the incidence of postoperative abdominal abscess in adult patients with complicated appendicitis [11].

Our study provides further evidence to this discussion by retrospectively analyzing patients who received an appendectomy for complicated appendicitis and received intraoperative irrigation and/or drainage. By analyzing a high volume of patients at an academic tertiary system, our study uniquely presents that intraoperative drainage in this group of patients could potentially be harmful by increasing the risk of postoperative abscess and sepsis. Our study therefore furthers the results obtained by Greenall et al and provides additional evidence that this surgical technique may actually be harmful.

Similar to evidence in current literature that supports the tenant that intraoperative irrigation provides nil benefit, our study further supports the perspective that irrigation has no benefit during complicated appendicitis cases.

Our study also demonstrates that both intraoperative drainage and/or irrigation increase length of hospital stay. This is significant when considering the benefit of these surgical techniques to overall patient care. By increasing the length of hospital stay, patients have increased cost of care, increased risk of hospital-acquired infections, and increased risk of resistance to commonly used antibiotics.

One major limitation of the study is it being retrospective in nature. Tables 1 and 2 demonstrate a statistically significant difference between irrigated versus not irrigated and drained versus not drained when it pertains to the following characteristics: presenting with generalized peritonitis, presenting with localized peritonitis, receiving an open appendectomy, and receiving a laparoscopic appendectomy. This raises the potential question if a patient's initial severity of illness could have affected both primary and secondary outcomes analyzed in this study. Given the retrospective nature of the study, however, this facet was hard to determine. Future studies should analyze the use of intraoperative drains and/or irrigation in complicated appendicitis cases with a prospective, randomized trial approach. By utilizing this approach, the limitations of this study could be further elucidated and accounted for.

\section{Conclusions}

Our study has demonstrated, retrospectively, that the use of intraoperative drains could potentially be harmful by increasing the risk of postoperative abscess and sepsis. The use of intraoperative drains and/or irrigation also increases the length of hospital stay. As clinical practice moves towards utilizing evidence-based guidelines and promoting higher-value health- care to patients, surgeons have to be critical of the surgical techniques and resources used. By utilizing the evidence established in this study to guide future studies, surgeons and clinicians can establish more concrete guidelines when it comes to the employment of intraoperative drains and/or irrigation for complicated appendicitis cases.

\section{Acknowledgments}

We would like to thank St. Joseph's University Medical Center.

\section{Financial Disclosure}

The authors report no proprietary or commercial interest in any product mentioned or concept discussed in this article.

\section{Conflict of Interest}

Authors report no conflict of interest.

\section{Informed Consent}

Not applicable.

\section{Author Contributions}

MS collected data and wrote the paper; MG conceived and designed the analysis; JM performed the analysis; PH contributed to data or analysis tools; JH edited the paper; JZ conceived and designed the analysis.

\section{References}

1. Smink D, Soybel D. (2018, October 1). UpToDate. Retrieved December 3, 2018, from https:/www-uptodatecom.proxy.libraries.rutgers.edu/contents/managementof-acute-appendicitis-in-adults? search=perforated appendicitis $§ i o n R a n k=2 \&$ usage type $=$ default\&anchor $=$ H18\&source $=$ machineLearning\&selectedTitle $=1 \square 32 \& d$ isplay_rank=1\#H18.

2. Schein M. To drain or not to drain? The role of drainage in the contaminated and infected abdomen: an international and personal perspective. World J Surg. 2008;32(2):312321.

3. Smith SR, Gilmore OJ. Surgical drainage. Br J Hosp Med. 1985;33(6):308, 311, 314-315.

4. Fischer JE, Jones DB, Pomposelli FB, et al. Fischers mastery of surgery. Philadelphia: Wolters Kluwer/Lippincott Williams \& Wilkins. 2012.

5. Magarey CJ, Chant AD, Rickford CR, Margarey JR. Peritoneal drainage and systemic antibiotics after appendicectomy. A prospective trial. Lancet. 1971;2(7717):179-182. 
6. Greenall MJ, Evans M, Pollock AV. Should you drain a perforated appendix? Br J Surg. 1978;65(12):880-882.

7. Beek MA, Jansen TS, Raats JW, Twiss EL, Gobardhan PD, van Rhede van der Kloot EJ. The utility of peritoneal drains in patients with perforated appendicitis. Springerplus. 2015;4:371.

8. Pakula AM, Skinner R, Jones A, Chung R, Martin M. Role of drains in laparoscopic appendectomy for complicated appendicitis at a busy county hospital. Am Surg. 2014;80(10):1078-1081.

9. St Peter SD, Adibe OO, Iqbal CW, Fike FB, Sharp SW, Juang D, Lanning $\mathrm{D}$, et al. Irrigation versus suction alone during laparoscopic appendectomy for perforated appendicitis: a prospective randomized trial. Ann Surg. 2012;256(4):581-585.

10. Hajibandeh S, Hajibandeh S, Kelly A, Shah J, Khan RMA, Panda N, Mansour M, et al. Irrigation versus suction alone in laparoscopic appendectomy: is dilution the solution to pollution? A systematic review and meta-analysis. Surg Innov. 2018;25(2):174-182.

11. Sun F, Wang H, Zhang F, Zhang X, Xing Z, Zhang S, Zhang $\mathrm{H}$, et al. Copious irrigation versus suction alone during laparoscopic appendectomy for complicated appendicitis in adults. J Invest Surg. 2018;31(4):342-346. 